\title{
Experimental Use of Kit-Build Concept Map System to Support Reading Comprehension of EFL in Comparing with Selective Underlining Strategy
}

\author{
Mohammad ALKHATEEB \\ Department of Information Engineering \\ Hiroshima University \\ Department of computer engineering \\ Tishreen University \\ Hiroshima, Japan \\ Yusuke HAYASHI \\ Department of Information Engineering \\ Hiroshima University \\ Hiroshima, Japan
}

\author{
Taha RAJAB \\ Institute for effective Education \\ University of York \\ York, \\ UK
}

Tsukasa HIRASHIMA

Department of Information Engineering

Hiroshima University

Hiroshima, Japan

\begin{abstract}
In this paper, we describe the effects of using KitBuild concept mapping (KB-mapping) method as a technologyenhanced support for the Reading Comprehension (RC) in English as Foreign Language (EFL) contexts. $\mathrm{RC}$ is a process that helps learners to become a more effective and efficient reader. It is an intentional, active and interactive activity that language learners experience in their daily working activities. RC of EFL is a significant research area in technology-enhanced learning. In order to clarify the effect of KB-mapping method, we compared the results with that of selective underlining (SU) strategy through the Comprehension Test (CT) and the Delayed Comprehension Test (DCT) that performed two weeks later. As the results, it is clarified that there is a noticed difference in the DCT scores, while there is no significant difference in the CT scores. It indicates that the use of KB-mapping method helps learners to retain their information for longer period of time. By doing more statistical analysis for the results of the Kit- Build Conditions (KB-conditions) group and comparing them with the map scores, we found that the learners could answer $76 \%$ of the questions whose answers were included in their learner's maps. It was found that learners could recall $86 \%$ of the questions and that their answers were included in their learner's maps. It indicates that the use of KB-mapping method helps learners to retain and recall more information compared with the $\mathrm{SU}$ strategy even after two weeks elapsed. In a follow-up questionnaire after the end of all experiments, it was revealed that participants thought that using KB-mapping was similar to SU for the CT just after the use, but KB-mapping was more useful in remembering information after a while, and it was more difficult to carry out. Participants liked to use it in RC tasks, but asked for more time to do it.
\end{abstract}

Keywords-Technology-Enhanced Learning; Kit-Build Concept Map; Reading Comprehension; EFL

\section{INTRODUCTION}

Reading comprehension (RC) is one of the important learning activities, and it needs a special ability for learners to reap its benefits [1]. RC poses many challenges such as slow reading, insufficient vocabulary comprehension and bad recalling [ HYPERLINK V "Bro81" 2 ]. Researchers have always tried to support this learning activity by proposing methods or strategies. The main goal is to boost comprehension skills in the target subject area. When they are deployed in a language course, the main aims are to improve student RC skills and to contribute to the acquisition of the target language 3].

The RC in an English as foreign language (EFL) context is a special case of RC; it is highly complex, dynamic, multicomponential and multi-dimensional task in the learning process. We can explain it as multiple interactions between the reader's background and knowledge in his or her Mother Language (ML) and that in English. Broadly speaking, the EFL $\mathrm{RC}$ is the same as the ML RC but it is slower and less successful than ML reading [ HYPERLINK \l "And99" 4 ]. This can be explained in that the reading process is depending on many factors such as the level of reader's language proficiency, type of text, text difficulty and task demands.

In general, RC ability of EFL readers are not enough because of their low language proficiency, low awareness of RC strategies, limited vocabulary knowledge and potential comprehension failure5] [ HYPERLINK 1 "Pan08" 6 ]. So we can consider them as poor readers.

In our previous researches, we confirmed that KB-mapping method has the same effects of Scratch Build concept Mapping (SB-mapping) method in the CT, just after the use of method, but KB-mapping method has better effects in recalling after two weeks7]. There are many researchers have confirmed that SB-mapping method is a very effective supporting method for EFL RC [ HYPERLINK \l "Man12" 8 ]9] [ HYPERLINK 1 "Sal13" 10 ]. Some researches have mentioned that SCmapping method had two weak points. Firstly, poor readers lack the ability to construct knowledge and seldom utilize 
learning strategies11] [ HYPERLINK \ "Lun95" 12 ]. Secondly, poor readers have weaker meta-cognitive ability and have difficulties associated with not being aware of the reading process13] [ HYPERLINK VI "Zen99" 14 ]. This means that SB-mapping method is not fit for EFL learners as poor readers. So, only comparing KB-mapping method with SB-mapping method is not enough. It is necessary to compare with reasonable method to support poor readers. Selective Underlining (SU) strategy is an authorized method that is useful for poor readers8] [ HYPERLINK VI "Cla14" 15 ]. So in this paper, KB-mapping method is compared with $\mathrm{SU}$ strategy as a supportive method for EFL learners.

In this paper, "our hypothesis is that KB-mapping method has the same effects as SU strategy for the short term, and is more effective for the long term as shown in our previous researches based on SB-mapping method". In order to proof this hypothesis, we conducted an experiment to compare with SU strategy as the previous one.

In general, for long term learning process, the improvement of the ability is not easy to investigate, also the investigation process requires a special environment to keep the fare of the results, as an example to prove that there is no other method or strategy was used by the learner, during the learning time, is impossible, so in this research, we investigated the performance of the learners during the learning process16].

\section{A. EFL Reading Comprehension}

The Comprehension is defined as "the ability to understand something" in the Oxford Dictionary, the definition of Cambridge Dictionary is "the ability to understand completely and be familiar with a situation, facts, etc.", from these two definitions, we can define the RC in our research as "the learner's ability to understand completely and memorize the important information that is included in the text, that he is reading". Also RC is defined as the level of understanding of a text/message. This understanding comes from the interaction between the words that are written and how they trigger knowledge outside the text/message [ HYPERLINK $\mid$ "And99" 4 ].

Fergus and Lockhart17] had proposed a theory that the RC involves two levels of processing, shallow (low-level) processing and deep (high-level) processing. This theory describes memory recall of stimuli as a function of the depth of mental processing. Deeper levels of analysis produce more elaborate, longer lasting, and stronger memory traces than shallow levels of analysis. Depth of processing falls on a shallow to deep continuum. Shallow processing (e.g., processing based on phonemic and orthographic components) leads to a fragile memory trace that is susceptible to rapid decay. Conversely, deep processing (e.g., semantic processing) results in a more durable memory trace. Many researches have used the memory recalling as indictor to measure the level of comprehension, and they have proposed the delayed comprehension test for the depth of comprehension [ HYPERLINK V "Par81" 18 ]19]. As an example, Mayer and Bluth [ HYPERLINK \ "Mey80" 20 ] have proposed to check the recalling immediately, and one week later. Due to this theory, we measured the level of RC by using two kinds of test, the Comprehending Test (CT), which was done just after the practical use, to check the comprehended information from the text (Shallow processing), and the Delayed Comprehension Test (DCT), which was done two weeks after, in order to measure the recalled information (Deep processing).

In general, the RC is a very difficult task for learners in all the stages of study especially when they are reading text in a foreign language. Thus, the EFL reading is one of the most common research topics in the learning field.

Many researchers have tried to solve this problem by proposing sstrategies to help the EFL learners in the RC task; SU strategy15] [ HYPERLINK V1 "Pio051" 21 ], Note-Taking Skills Reading21], SQ3R (Survey, Question, Read, Recite, Review) [ HYPERLINK VI "Hub04" 22 ] and PORPE (Predict, Organize, Rehearse, Practice, Evaluate)23]. However, most of them only had slight improvements in the EFL learners' comprehension just after the use. Several investigations of RC strategies have specifically addressed challenges related to reading expository text. Positive outcomes have been found for learners who were taught strategies to help learners identify main ideas [ HYPERLINK 11 "Pan08" 6 ].

\section{B. Selective Underlining Strategy}

SU strategy is one of the most important strategies commonly used in classroom. Generally, it is used to help learners to organize the text, which they are reading by selecting the important sentences. This strategy teaches learners to highlight/underline only the key words, phrases, vocabulary, and ideas that are essential to understanding the text15] [ HYPERLINK \ "Hub04" 22 ]. It is very useful for comprehending the text, because it is a flexible strategy that may be tailored to fit various types of information and different skill-levels. This strategy can also be integrated with the use of technology and electronic information such as eBooks. As students study, it helps them learn to pay attention to the essential information within a text15]. In general, this strategy is focusing on the vocabularies and comprehension of the text during the reading time, also it helps the learners to identify the important points in text, helps them to pay close attention to what they are reading and also, allow greater learning and deeper comprehension.

Typically, in the SU strategy, the learner starts by reading the text to understand the main content of the text, after that, the learner rereads and begins to underlining the main ideas and their supporting details, then he selects the important facts and the key vocabularies [ HYPERLINK \ "Cla14" 15 ].

By using the underlined part of the text, the learner can give a summary for the important information in the text that he has read. This strategy demands the learner to capture the main ideas, key concepts and details; also it helps learning by reducing the needed information in text, so it reduces the learning time and in the same time strengthens the RC.

As SU strategy is widely used in usual classroom, and it is suitable strategy to support the poor readers, as the learners of EFL RC. So we planned to compare KB-mapping method with $\mathrm{SU}$ strategy in order to confirm the usability of $\mathrm{KB}$-mapping method for supporting EFL poor reader too. 


\section{KB-MAPPING METHOD}

We have been developing learning tools to help both the students and the teachers in the learning process. One of these tools is KB-Map24] [ HYPERLINK VI "Yam10" 25 ]26], and we have found the following results; (1) this tool is very useful for learning different topics of sciences in learner's mother languages [ HYPERLINK \ "Sug12" 27 ]28], and (2) this method has the same effect of SB-mapping method just after the building task, but it has better effects in recalling after a while [ HYPERLINK \1 "Alk15" 7 ]16].

\section{A. Overview of KB-map (Kit-Build Concept Map)}

As a definition of KB-map we can find "a framework to realize automatic diagnosis of concept maps built by learners and to give feedback to their errors in the maps" [ HYPERLINK V "Yam10" 25 ]. KB-map is a special kind of concept map. Generally, the concept map creation consists of two steps; (1) the extraction of the concepts and the relations from a specified text, and (2) the making-connection between two extracted concepts by using the extracted relation. In KBmapping method, the supervisor of learners performs the first step to create the goal map, and generates a kit by dividing the goal map. Then, the learner is asked to make connections by using the kit until he or she connects all of components in the kit.

\section{B. KB-mapping System}

We have already developed a system based on the KB-map explained in the previous section. This system is called "KBmapping System". It is a web-based application with two client systems: "KB-map Editor" and "KB-map Analyzer", and a server system "KB-map DB". KB-map Editor provides an environment for teacher, or supervisor, to make a goal map, a kit, and for learner to make a learner's map. This system has been implemented by Java (version 1.6). KB-map Analyzer has functions to gather learner's maps online, generate a group map and diagnose the maps. This system has implemented by Flash and is used with Flash Player 10. KB-map DB has a function to store and share maps. This system has been developed by Ruby (version 1.8.7) on Rails (version 1.2.3) and MySQL (version 5.1.30)25].

\section{EXPERIMENT METHODOLOGY}

We investigate the effects of the KB-mapping method as a supportive tool for RC task using the CT for the short term and the DCT for the long term described in Section X, by comparing with those of the SU strategy under the same conditions. In this section, we describe the following four points of experiment; (1) the subjects, (2) the procedure of RC task, (3) the learning materials and (4) the experimental system.

\section{A. The subjects}

The subjects of our experiment were eight Japanese students of third grade of information engineering faculty. Their scores of TOEIC exam vary from 430 625, so they had different reading abilities; we prepared an aptitude test to check their reading abilities, this test was a simple reading test in the same level of the used in all the sessions. By using the information of their TOEIC records and aptitude test results, we grouped them into two groups $\mathrm{A}$ and $\mathrm{B}$, which are almost commensurate with the reading ability as shown in Table I.

TABLE I. PARTICIPANTS GROUPING

\begin{tabular}{|l|l|l|}
\hline & Group A & Group B \\
\hline TOEIC (SD) & $513.75(75)$ & $537.5(71)$ \\
\hline Aptitude Test & 70 & 65 \\
\hline
\end{tabular}

\section{B. Procedure of Experiment}

We performed this experiment in six sessions as RC task for six different English texts; At the beginning of the first session, we gave instructions for subjects about the procedure of experiments including how to use KB-mapping system. As for the rest of sessions, we started with the DCT of the previous session, followed by a learning activity for thirty minutes in which subjects tried to improve their English level. At the end of the sixth session, we asked subject using questionnaire described later.

\section{Procedure of One Session:}

In our experimental use, we compared the effects of using KB-mapping method and the effects of SU strategy in the RC process. We were measuring these effects from two points of view, just after using and two weeks later.

To eliminate the effect of other supporting strategies, we designed our experiment learning activity under strict and limited time constrains. The process of one session, as shown in Table II, consists of 4 steps: Firstly, within 10 minutes, the both conditions groups were requested to comprehend the whole text by reading it generally and translating the difficult words in the text by using dictionary, then in the next 10 minutes the $\mathrm{KB}$-conditions group was required to build the $\mathrm{KB}$-map of the text by using KB-map editor and in the same time the SU-conditions group was required to do the underlining of the important sentences in the text. After that both groups did the CT within 5 minutes, and finally, after two weeks both groups did the CT again as a DCT.

TABLE II. PROCEDURE OF ONE SESSION

\begin{tabular}{|l|l|l|}
\hline time & KB-conditions & SU-conditions \\
\hline $10 \mathrm{~min}$ & \multicolumn{2}{|l|}{ Reading materials (using dictionary is allowed) } \\
\hline $10 \mathrm{~min}$ & $\begin{array}{l}\text { Making the KB-map by } \\
\text { using KB-map editor }\end{array}$ & $\begin{array}{l}\text { Underlining the important } \\
\text { parts of the text }\end{array}$ \\
\hline $5 \mathrm{~min}$ & Doing Comprehension Test \\
\hline $5 \mathrm{~min}$ & \multicolumn{2}{|l|}{ Doing DCT(2 weeks after) } \\
\hline
\end{tabular}

\section{EXPERIMENTAL USE}

This experiment was done in 6 sessions with two groups of participants (A, B) both of them had almost the same reading ability. For each group they used the KB-mapping method for 3 sessions and the SU strategy for 3 sessions too. To conduct this experiment we used computers with Intel core i3-3240 processor, $4 \mathrm{~GB}$ of RAM and $20^{\prime \prime}$ monitor. The used platform was windows 7 . In this chapter we introduced 3 points: the preparation of the used materials, example of the real experimental use and example of the materials that used in the experiment. 


\section{A. Materials Preparation}

The participants of this experiment were information engineering students, they were interested in the topics of information engineering, so firstly we selected 6 text from Wikipedia in the information engineering field, and checked their grammatical and semantic structure. We created the corresponding KB-maps (Goal maps) that covered the main concepts and relations of the texts, also we did the SU for the important and essential phrases in the texts. Then we prepared the CTs. Finally, we checked all of the material again to make sure that materials did not contain any error. In addition, we checked if the answers of questions of CT were included or not in the concepts extracted by the KB-mapping method and the SU strategy, to marking the questions that not covered.

\section{B. Example of the experimental use}

To investigate the effects of using KB-mapping method to improve the EFL CT, For the first session, we started with a learning activity to improve the English level of student, and in the last 30 minutes we did our experiment, firstly, within ten minutes, the learners were requested to read the text to get the main idea of it and to translate the unknown words for them by using a web dictionary. After that within 10 minutes too, the KB-conditions group tried to build the learner's map by using the KB-map editor, in the same time, the SU-conditions group were doing the SU of the important phrases of the text. In the next 5 minutes they answered the CT. Finally we collected all the materials about this session (text, notes and test papers). Two weeks after, the participants did the DCT. Figure2 shows the process of the first session.

For the other sessions, they did the DCT of the previous session, as we mentioned before, and after that, they started the new session as explained in the previous paragraph.

\section{Example of experimental material}

In this section, we introduce one example of the materials that was used in the first session of our experiment. Fig. 1 shows a part of the text that was used as the original text, which learners tried to comprehend it. After that, the KBconditions group tried to build learner's map, within 10 minutes, by using the kit that provided by the system, which generated from the corresponding goal map. Fig. 3 shows the goal map of the text that was prepared by the supervisor. It contains most of the information of the original text, this goal map was divided by the system to generate the kit that shown in Fig. 4. Fig. 5 shows one example of the learner's map which was built by one of the learners.

In the same time, the SU-conditions group tried to do the $\mathrm{SU}$ for the important sentences in the text within 10 minutes too. The underlined text contains the important information of the text. Fig. 2 shows one example of the underlining of the same paragraph.

"A general purpose computer has four main components: the arithmetic
logic unit (ALU), the control unit, the memory, and the input and output
devices. These parts are interconnected by busses, often made of groups of
wires.
Inside each of these parts are thousands to trillions of small electrical
circuits which can be turned off or on by means of an electronic switch. The
circuits are arranged in logic gates so that one or more of the circuits may
control the state of one or more of the other circuits."

Fig. 1. Example, Part of First Session's Text
"A general purpose computer has four main components: the arithmetic logic unit (ALU), the control unit, the memory, and the input and output devices. These parts are interconnected by busses, often made of groups of wires.

Inside each of these parts are thousands to trillions of small electrical circuits which can be turned off or on by means of an electronic switch. The circuits are arranged in logic gates so that one or more of the circuits may control the state of one or more of the other circuits."

Fig. 2. Example, First Session's Underlined Text

After that all the learners did the same CT, that lasted for 5 minutes and which was a set of multi-choices questions scored from 100. All of these questions were asking about information included in the original text, some of them were asking about information included in the goal map. For the underlined text all of them were included. Fig. 6 shows a part of the CT of this session.

By the end of this test, they have finished the experimental use of that day and 2 weeks later, they did the comprehension test again as a DCT.

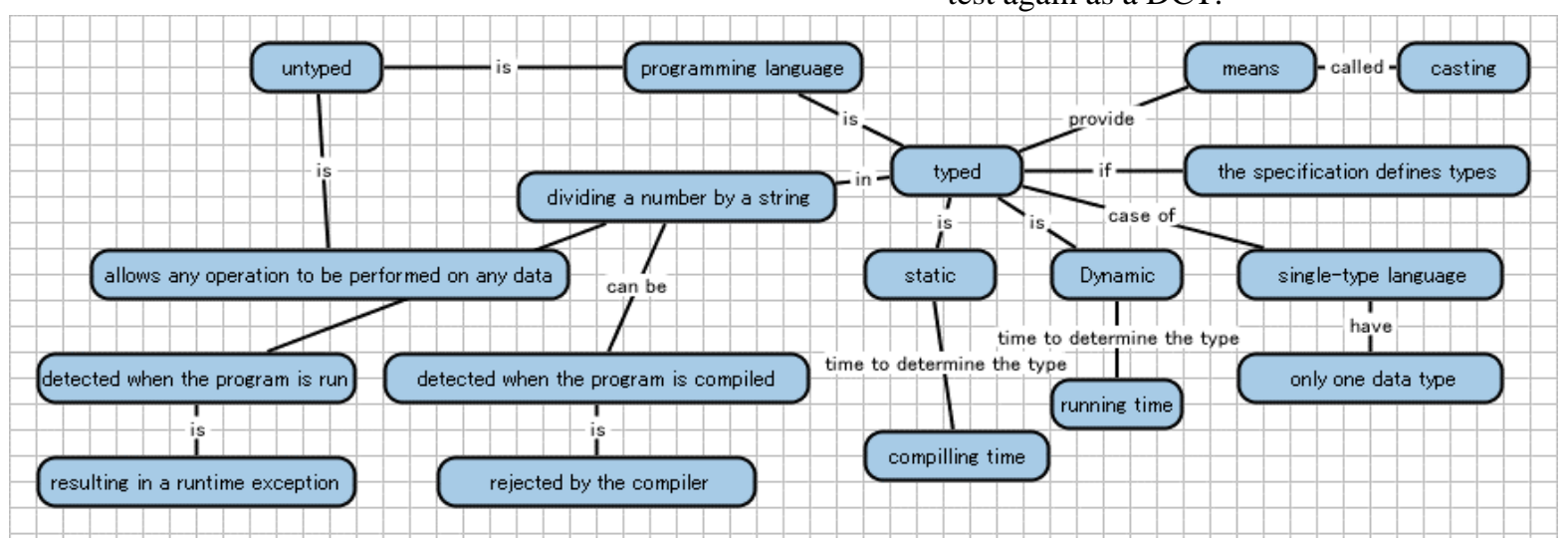

Fig. 3. The Goal Map of First Session 


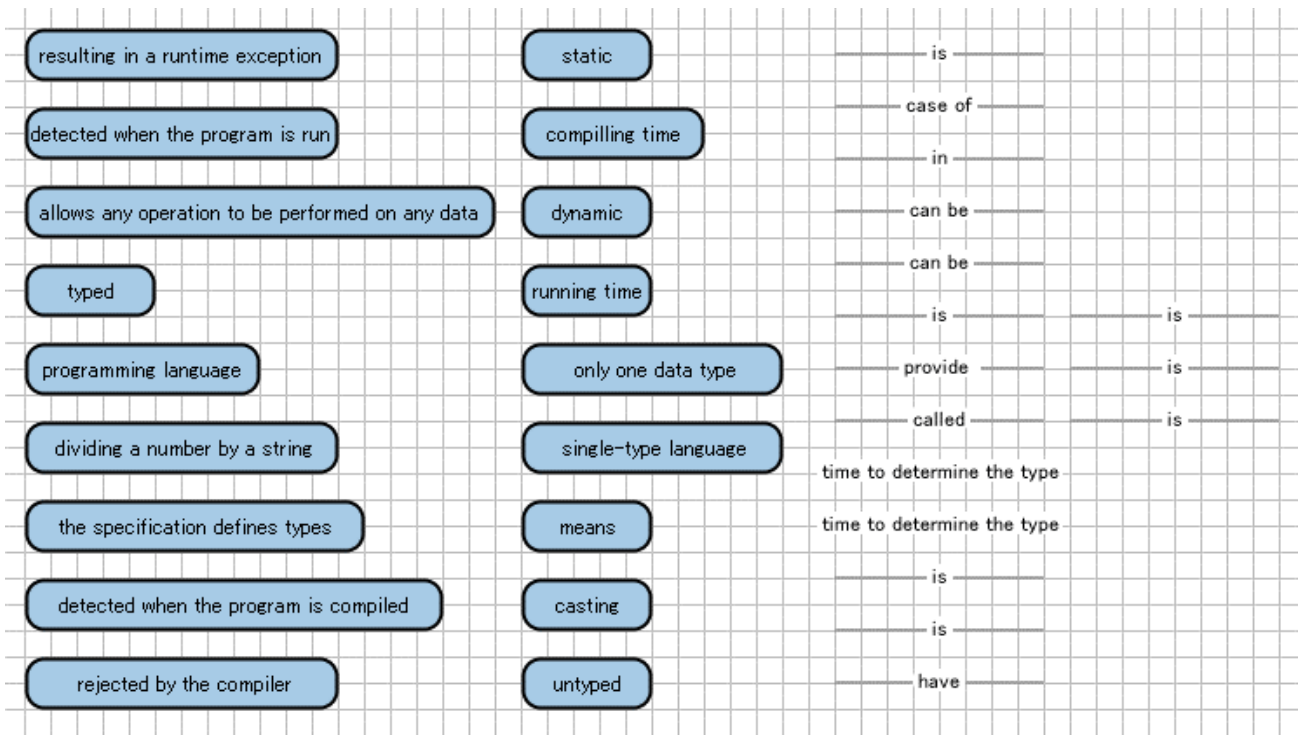

Fig. 4. The Kit of firest session

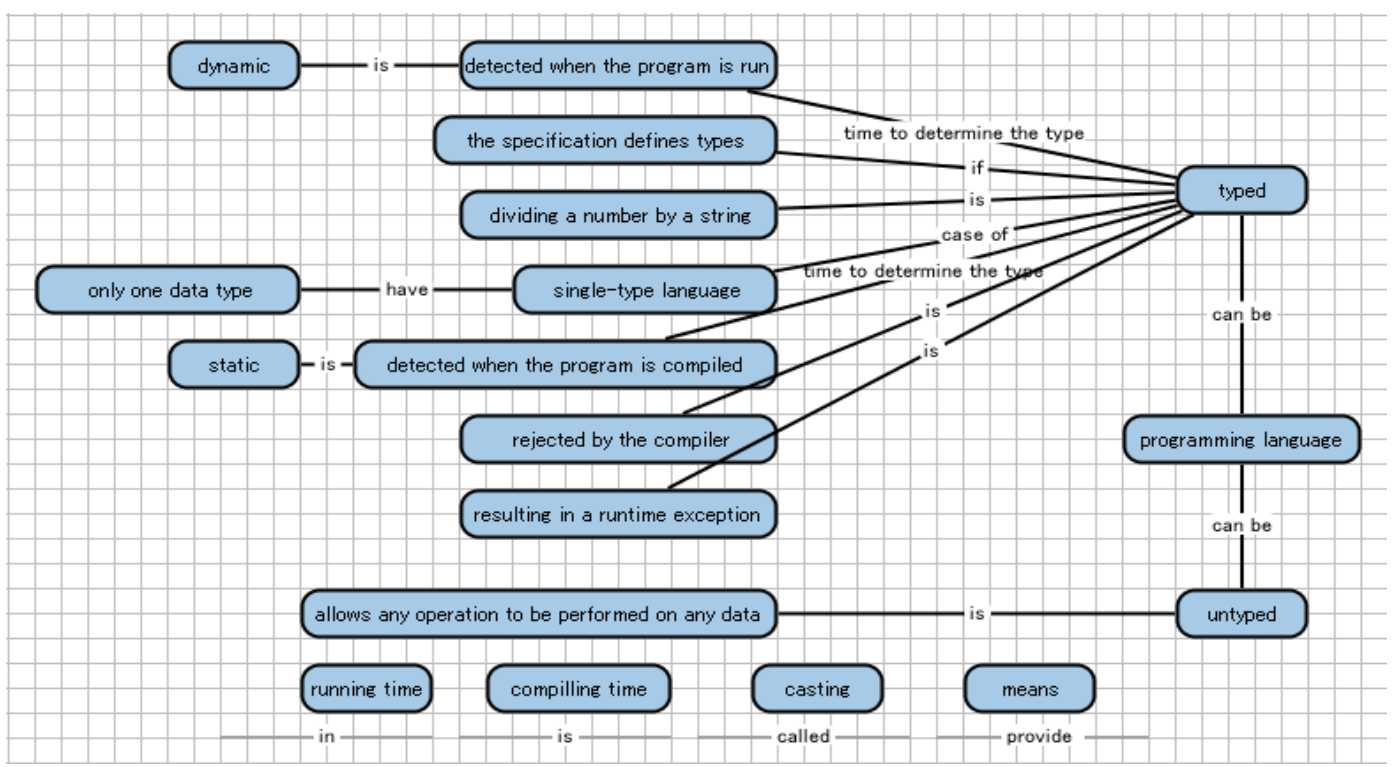

Fig. 5. Example of the learner's map

Q3: What is the component of busses?
a. Electrical circuits
b. Logical gates
c. Wires
d. Wires and logical gates

Q4: What are inside the control unit?
a. Electrical circuits
b. Logical gates
c. Wires
d. Electrical circuits and logical gates

Q5: what can the electric circuits control?
a. Electrical circuits
b. Logical gates
c. Wires
d. The control Unite

Fig. 6. Sample of the questions in comprehension test

\section{RESULTS}

We performed our experiment with 8 students in 6 sessions. In the first session, we had 4 participants as the KB-conditions (Group A) and 4 as the SU-conditions (Group B), then the participants were shifted to the alternate conditions in the next and so on. The groups are made by balancing in the scores of TOEIC as shown in Table1. We had 6 sessions with different 6 texts. Goal maps and tests are prepared for each text. Table III summarizes the details of every session.

TABLE III. DETAILS OF EVERY SESSION

\begin{tabular}{|l|l|l|l|l|l|l|}
\hline Session No. & $\mathbf{1}$ & $\mathbf{2}$ & $\mathbf{3}$ & $\mathbf{4}$ & $\mathbf{5}$ & $\mathbf{6}$ \\
\hline KB-Conditions & Group A & B & A & B & A & B \\
\hline SU-Conditions & Group B & A & B & A & B & A \\
\hline Text & T1 & T2 & T3 & T4 & T5 & T6 \\
\hline
\end{tabular}




\section{A. Effects of Using KB-mapping Method in CT and DCT}

For every session, we compared the CT average scores, the DCT average scores and the differences (DCT-CT) for the two conditions group. The scores are shown in Table IV. We found that the $\mathrm{KB}$-conditions have a better $\mathrm{CT}$ average score in one session and nearly worse average in the remaining five. For the DCT, we found that the KB-conditions have a better DCT average score in five sessions and nearly worse average scores in the remaining one. And for the difference DCT-CT (Diff), we found that the KB-conditions have a higher average in all the sessions.

TABLE IV. AVERAge COMPREHENSION TEST \& DELAYED COMPREHENSION TEST SCORES FOR THE TWO CONDITIONS

\begin{tabular}{|l|l|l|l|l|l|l|}
\hline Session No. & $\mathbf{1}$ & $\mathbf{2}$ & $\mathbf{3}$ & $\mathbf{4}$ & $\mathbf{5}$ & $\mathbf{6}$ \\
\hline KB-CT & 80 & 42.5 & 50 & 62 & 60 & 80 \\
\hline SU-CT & 75 & 80 & 41 & 87 & 65 & 90 \\
\hline KB-DCT & 90 & 55 & 65 & 58 & 65 & 70 \\
\hline SU-DCT & 65 & 60 & 45 & 45 & 35 & 55 \\
\hline
\end{tabular}

In the remaining one case where KB-condition is worse than SU-condition in DCT, we found that the learners failed to complete a concrete learner's maps in comparison with other cases. As they could only get about $37 \%$ as a map score, in comparison with the other session average was $74 \%$, because of the complexity of goal map (map size was big) and the limitation of time (10 minutes only for building). In general when learners failed to build learners' map they could not comprehend the whole text as structured form. This may be case a misunderstanding for the information.

In this experiment, the two groups of subjects are balanced by their TOEIC scores and aptitude test scores, both groups had followed KB-conditions for three times and SU conditions for three times too. The process of KB-conditions and SUconditions were same in all phrases except the phrase of building learner's map and doing the SU for the text, which had the same time to do. We analyzed the data by using winlose-tie statistical analyze to show which is better among the CT, DCT and the difference (Diff=DCT-CT). Table V is the summary of the win-lose data.

TABLE V. Win-Lose TABLE AND BinOMINAL TeSt OF THE KBCONDITIONS SCORES

\begin{tabular}{|c|c|c|c|c|c|c|c|c|}
\hline Session No. & $\mathbf{1}$ & $\mathbf{2}$ & $\mathbf{3}$ & $\mathbf{4}$ & $\mathbf{5}$ & $\mathbf{6}$ & $\sum$ & $\boldsymbol{P}$ (value) \\
\hline CT & 1 & 0 & 1 & 0 & 0 & 0 & 2 & 0.234 \\
\hline DCT & 1 & 0 & 1 & 1 & 1 & 1 & 5 & 0.094 \\
\hline Diff & 1 & 1 & 1 & 1 & 1 & 1 & 6 & 0.016 \\
\hline
\end{tabular}

To evaluate the results of the win-lose-tie table, we used the binominal test to calculate the probability mass function of the number of KB-mapping method winnings in all the sessions. By using the binominal test, We found that the KB-conditions won in the CT for 2 of six sessions ( 2 wins +4 lose); the probability that there were two successes in six trials was ( $p$ $(2 / 6)=0.234>0.05)$. This result indicated that the KB-mapping method did not show better effects in the CT just after being used. For the DCT, we found that the KB-conditions won in five of six sessions ( 6 wins); the probability that there were 5 successes in six trials was $(p(5 / 6)=0.094>0.05)$; This was slightly significantly different $(p<0.10)$; this result indicated that the KB-mapping method showed better effects in the DCT two weeks after being used. Also for the differences (DCT$\mathrm{CT}$ ), we found that the KB-conditions won in six of six sessions (six wins), and the probability that there were six successes was $(p(6 / 6)=0.016<0.05)$; this probability was significantly different. This result indicated that the KBmapping method had better effects on recalling the comprehended information 2 weeks after being used.

\section{B. Effects of Using KB-mapping Method in CT and DCT}

To investigate the effects of using KB-mapping method, we did more detailed analysis about the included questions in learner's map of our experiment.

The included questions were the ones that can be answered by using the learner's map, as example, in Fig. 6 the questions 3 and 4 were included in the learner's map that shown in Fig. 5 , they could be answered by using the components of the learner's map.

For the CT, we calculated the score of the included questions, that their answers were correct, in the learner's map and compared them with the total number of the included questions in the learner's map. We found that the KBconditions learners could answer $76 \%$ of the questions that their answers were included in their learner's maps.

$$
A v\left(\frac{\text { correct answer in comprehension test included }}{\text { included questions in learner's map }}\right) * 100=
$$

In (1), we calculated the number of the correct answered questions in the $\mathrm{CT}$, which their answers were included in the learner's map and compared them with the total number of questions, which their answers were included in the learner's map, finally we calculate the average of all learners.

For the DCT, we calculated the score of the included questions in the learner's map and compared them with the total number of the included questions. We found that the KBconditions learners could remember $86 \%$ of the questions that their answers were included in their learner's maps.

$$
\begin{gathered}
\mathrm{Av}\left(\frac{\text { correct answer in delayed comprehension test included }}{\text { included questions in learner's map }}\right) * \\
100=85.625 \text { (2) }
\end{gathered}
$$

In (2), we calculated the number of the correct answered questions in the DCT, which their answers were included in the learner's map and compared them with the total number of questions which their answers were included in the learner's map, finally we calculated the average.

From the (1)\&(2), we could say that the using of KBmapping method helped the learners to use and recall, after two weeks, most of the information that was included in their learner's map.

\section{Effects of Text Without KB-map in CT and DCT}

To ensure the reliability of our findings about the effects of using KB-mapping method, by checking the effects of the other parts of the text that were not included in the learner's map, we did more detailed analysis about the questions that 
were not included in learner's map called as "not included questions".

The "not included questions" were the questions that cannot be answered by using the learner's map, as example, in Fig. 6 the questions 5 is not an included questions in the learner's map is shown in Fig. 5, they could not be answered by using the components of the learner's map.

For the CT, we calculated the score of the" not included questions" that their answers were correct, in the learner's map and compared them with the total number of the "not included questions". We found that the KB-conditions learners could answer only $45 \%$ of the questions that were not included in their learner's maps.

$$
\operatorname{Av}\left(\frac{\text { correct answer in comprehension test not included }}{\text { not included questions in learner's map }}\right) * 100=
$$

In (3), we calculated the number of the correct answered questions in the CT which their answers were not included in the learner's map and compared them with the total number of questions which their answers are not included in the learner's map", finally we calculate the average.

For the DCT, we calculated the score of the "not included questions" in the learner's map and compared them with the total number of the not included question. We found that the KB-conditions learners could remember only $42 \%$ of the questions that were not included in their learner's maps.

$\operatorname{Av}\left(\frac{\text { correct answer in delayed CT not included }}{\text { not included questions in learner's map }}\right) * 100=42.0139$

In (4), we calculated the number of the correct answered questions in the DCT which their answers were not included in the learner's map and compare them with the total number of questions which their answers were not included in the learner's map, finally we calculated the average.

From the (3)\&(4), we could say that the effects of the text without KB-mapping method was not so helpful for the learners to answer or to remember the information that not included in their learner's maps.

As a summarization for section 5.3 and 5.4, we found that if the learner could build a good learner's map, he would get a good score in the CT and he would get a good score in the DCT. In other words, the learner's map could be used to evaluate the learner's comprehension.

\section{Questionnaire}

After we had finished the last session's DCT, the learners answered the questionnaire to evaluate the learning method of using KB-mapping method and compare it with the SU strategy. Table VI shows the results of this questionnaire.

The questions (1-6\&9) were multiple choice questions with 5 options that measure the participants agreement with the mentioned point by the question. The choices that used were "A. Strongly agree, B. Agree, C. Natural, D. Disagree and E. Strongly Disagree", and the questions (7\&8) were multiple choice questions with 3 options, that compare between the two learning methods. The choices that used were "A. SU, B. Same, C. KB-mapping"; to normalize the results of this questionnaire, we tried to summarize all the results of our questionnaire and convert them to arithmetical form that means (1 Strongly agree, 0.5 Agree, 0 Natural, $-0.5 \%$ Disagree, and -1 strongly Disagree) and in the same time. It is means (1 Map building, 0 same and -1 underlining). As a summarization of the questionnaire evaluation, 0 means the normal, the positive means agreement and the negative means disagree and the value shows the strength of the agreement or the disagreement.

From questions $(1 \& 4 \& 7)(2 \& 5)(3 \& 6)$, the learners found out that the KB-mapping method was useful to understand English text as the SU strategy and also useful to answer the $\mathrm{CT}$ just after the learning activity, but they thought that KBmapping method was more useful to answer the DCT two weeks after, Also from questions $(8,9)$ they thought that the KB-mapping method was more difficult to carry out, but they liked to use KB-mapping method in RC task but they mentioned that they need more time to do it.

\section{TABLE VI. Evaluation OF KB-MAPPING METHOD FOR EFL READING COMPREHENSION BY COMPARING WITH SU STRATEGY}

\begin{tabular}{|l|l|}
\hline Explanation & $\begin{array}{l}\text { Average } \\
\text { Agreement }\end{array}$ \\
\hline $\begin{array}{l}\text { Q1. Do you think that SU strategy was useful to understand } \\
\text { English text? }\end{array}$ & 0.1875 \\
\hline $\begin{array}{l}\text { Q2. Do you think that SU strategy was useful to answer the } \\
\text { test after reading? }\end{array}$ & 0.1875 \\
\hline $\begin{array}{l}\text { Q3. Do you think that SU strategy was useful to answer test } \\
\text { two week later? }\end{array}$ & -0.375 \\
\hline $\begin{array}{l}\text { Q4. Do you think that KB-mapping method was useful to } \\
\text { understand English text? }\end{array}$ & 0.5 \\
\hline $\begin{array}{l}\text { Q5. Do you think that KB-mapping method was useful to } \\
\text { answer the test after reading? }\end{array}$ & 0.375 \\
\hline $\begin{array}{l}\text { Q6. Do you think that KB-mapping method was useful to } \\
\text { answer test two week later? }\end{array}$ & 0.125 \\
\hline $\begin{array}{l}\text { Q7. Do you think that KB-mapping method was more useful } \\
\text { to understand English text? }\end{array}$ & 0.625 \\
\hline $\begin{array}{l}\text { Q8. Do you think that KB-mapping method was more difficult } \\
\text { to carry out? }\end{array}$ & 0.75 \\
\hline $\begin{array}{l}\text { Q9. Did you like to use KB-mapping method to understand } \\
\text { English text? }\end{array}$ & 0.0625 \\
\hline
\end{tabular}

\section{CONSIDERATION}

We found that the KB-mapping method user could retain the knowledge as the underlined method users, but they are more effective in the recalling. This result is harmonized with principle of structured storing of knowledge in memory, which proposed by cognitive psychology research. It has shown that the knowledge in the memory is stored in a structured form that determines the ability to retain, recall and use it to solve problems [ HYPERLINK \l "JMi01" 29 ]30] [ HYPERLINK Vl "Ohn11" 31 ]. For KB-mapping method, the learner tried to build the KB-map, which is a structured form of the knowledge, by using the provided information from the original text.

In general, the use of KB-mapping method needs concentration in reading the text and needs to read with attention to distinguish the two concepts, which can be related, 
and to find the corresponding relation, which can connect them together. In the same time, this process requires the learner to understand the information in the text deeply and required him to comprehend the text in whole. So we can explain our result by the required high load on the learner memory to comprehend deeply the whole text to complete the learner's map, this load forces the learner's memory to keep most of the information that he has already comprehended.

When constructing a KB-map, the focus is on the relationships among concepts, because the learner does not required to think about distinguishing the concepts that are already provided by the system, so his entire constraining is on the understanding of the whole text.

\section{CONCLUSION AND FUTURE WORK}

In this paper, we describe the effects of using KB-mapping method as a supportive tool for the RC of English texts as EFL reading. We conducted an experiment composed of six experimental RC sessions. Overall, from this experiment we can say that the use of KB-mapping as learning supportive tool for RC is good as SU strategy in the short term, but it is so better for the long term.

Our next step goal is to design a new experiment to compare our KB-mapping method with the Scratch Build Concept mapping method for $\mathrm{RC}$ supporting to investigate in deeply why KB-mapping method is more useful for recalling after a while.

\section{REFERENCES}

[1] M. F. Graves, F. Michael, J. Connie, and B. B. Graves, Teaching reading in the 21st century. Order Processing, Des Moines, 1998.

[2] A. L. Brown, J. C. Campione, and J. D. Jeanne, "Learning to learn: on training students to learn from texts", Journal. of Educational researcher, pp. 14-21, 1981

[3] K. Rayner, B. R. Foorman, C. A. Perfetti, D. Pesetsky and M. S. Seidenberg, "how psychological science informs the teaching of reading", Journal. of Psychological Science in the Public Interest, Vol. 2, No 2, pp. 31-74, 2001.

[4] N. J. Anderson, and X. Cheng, Exploring second language reading: Issues and strategies, MA: Heinle \& Heinle, Boston 1999.

[5] M. Zoghi, Masoud, M. Ramlee, and T. N. R. B. T. Mohamad "getting to know L2 poor comprehenders", Journal. of English Language Teaching, vol. 4, no. 1, pp. 98, 2011.

[6] J. Pang, "Research on good and poor reader characteristics: implications for L2 reading research in china", Journal of Reading in a Foreign Language, vol. 20, no.1, pp. 1-18, 2008.

[7] M. Alkhateeb, Y. Hayashi, T. Rajab, and T. Hirashima, "Comparison between kit-build and scratch-build concept mapping methods in supporting EFL reading comprehension", Journal of Information and Systems in Education, vol. 14, no.1, pp. 13-27, 2015.

[8] P. Manoli, and M. Papadopoulou.: "Graphic organizers as a reading strategy: research findings and issues", Journal of Creative Education,vol. 3, no.03, pp. 348 -356, 2012.

[9] M. Kalhor, and G. Shakibaei, "Teaching reading comprehension through concept map", Journal of Life Science Journal, vol. 9, no. 4, pp. 725-731, 2012.

[10] A. D. Salehi, S. Jahandar, and M. Khodabandehlou, "The impact of concept mapping on EFL student's reading comprehension", Journal of Indian J. of Fundamental and Applied Life Sciences,vol. 3, pp. 241-250, 2013.
[11] D. P. Clark, "first, poor readers lack the ability to construct", Journal of Dissertation Abstracts International, vol. 46, no. 8, pp. 22-46, 1985.

[12] I. Lundberg, "The computer as a tool for remediation in the education of students with reading disabilities: A theory based approach", Journal of Learning Disabilities Quarterly, vol. 18, pp. 89-100, 1995.

[13] R. Wagner, and J. K. Torgesen, "The nature of phonological processing and its causal role in the acquisition of reading skill", Journal. of Psychological Bulletin, vol. 101, pp. 192-212, 1987.

[14] S. J. Zeng, "Research of low achievement schoolchild in their work memory, the phonetic handling ability and the speed of reading", Proc. of the diagnosis of schoolchild's reading difficulty, pp. 5-28, taiwan1999.

[15] Schnell, R. Thomas, and J. R. Daniel, "a comparison of underlying strategies for improving reading comprehension and retention", Journal of Reading Horizons, vol. 18, no 2, pp. 106-109, 1978.

[16] M. Alkhateeb, Y. Hayashi, and T. Hirashima, "the effects of using kitbuild method to support reading comprehension of EFL" Proc. of Human Interface and the Management of Information. Information and Knowledge in Applications and Services, Springer International Publishing, pp. 3-11, 2014.

[17] F. I. Craik, and R. S. Lockhart, "Levels of processing: A framework for memory research", Journal of verbal learning and verbal behavior, vol. 11, no 6, pp. 671-684, 1972.

[18] S. G. Paris, and M. Myers, "Comprehension monitoring, memory, and study strategies of good and poor readers", Journal of Literacy Research, vol. 13, no 1, pp. 5-22, 1981.

[19] B. Laufer, "Vocabulary acquisition in a second language: Do learners really acquire most vocabulary by reading? Some empirical evidence", Journal of Canadian modern language review, vol. 59, no 4, pp. 567-587, 2003.

[20] B. J. Meyer, D. M. Brandt, and G. J. Bluth, "Use of top-level structure in text: key for reading comprehension of ninth-grade students", Journal of Reading research quarterly, pp. 72-103, 1980.

[21] A. Piolat., T. Olive. and T. Ronald "Cognitive effort during note taking", Journal of Applied Cognitive Psychology, vol. 19, no 3, pp. 291-312, 2005.

[22] J. A. Huber "A closer look at SQ3R", Journal of Reading Improvement vol. 4, no. 2, pp. 108-112, 2004.

[23] B. L. Ngovo, "Study strategies for narrative texts: PORPE and annotation", Journal of Developmental Education, vol. 23 no. 2, pp. 2428, 1999.

[24] H. Funaoi, K. Ishida, and T. Hirashima, "comparison of kit-build and scratch-build concept mapping methods on memory retention", Proc. of ICCE 2012 , pp. 539-546, 2012.

[25] K. Yamasaki, H. Fukuda, T. Hirashima, and H. Funaoi, "Kit-Build concept map and its preliminary evaluation", Proc. of the 18th International Conference on Computers in Education , pp. 290-294, 2010.

[26] T. Hirashima, K. Yamasaki, and H. Fukuda, "Framework of kit-build concept map for automatic diagnosis and its preliminary use", Journal. of Research and Practice in Technology Enhanced Learning, vol. 10, no. 1, pp. 1-21, 2015.

[27] K. Sugihara, T. Osada, S. Nakata, H. Funaoi, and T. Hirashima, "Experimental evaluation of kit-build concept map for science classes in an elementary school", Proc. of ICCE 2012, pp. 17-24, 2012.

[28] K. Yoshida, K. Sugihara, Y. Nino, M. Shida, and T. Hirashima, "Practical use of kit-build concept map system for formative assessment of learners", Proc. of ICCE 2013 , pp. 906-913, 2013.

[29] J. Michael, "In pursuit of meaningful learning", Journal of Advances in physiology education, pp. 145-158, 2001.

[30] R. C. Atkinson, and R. M. Shiffrin, "Human memory: A proposed system and its control processes", Journal of The psychology of learning and motivation, vol 2, pp. 89-195, 1968.

[31] T. Ohno, T. Hasegawa, T. Tsuruoka, K. Terabe, J. K. Gimzewski, and M. Aono, "Short-term plasticity and long-term potentiation mimicked in single inorganic synapses", Journal of Nature materials, vol.10, no. 8, pp. 591-595, 2011. 